STEADY-STATE ENZYME KINETICS 


\section{STEADY-STATE ENZYME KINETICS}

STANLEY AINSWORTH

Department of Biochemistry

University of Sheffield 


\section{(C) Stanley Ainsworth 1977}

Softcover reprint of the hardcover 1st edition 1977 978-0-333-15008-5

All rights reserved. No part of this publication may be reproduced or transmitted, in any form or by any means, without permission.

First published 1977 by

THE MACMILLAN PRESS LTD

London and Basingstoke

Associated companies in New York Dublin

Melbourne Johannesburg and Madras

ISBN 978-1-349-01961-8 ISBN 978-1-349-01959-5 (eBook)

DOI 10.1007/978-1-349-01959-5

Filmset in Ireland by Doyle Photosetting Ltd., Tullamore, Co. Offaly 


\section{ERRATA}

1. Page 4, line 9. Substitute 'thereby' for 'hereby'.

2. Page 8 , equation 1.5. Delete the three stray arrowheads in the lower centre part of the equation.

3. Page 37, equation 2.37. In the extreme R.H.S. of the equation substitute $E_{1}+P$ for $E_{0}+P$.

4. Page 57, equation 3.51. The R.H.S. numerator should read $V_{\mathrm{f}} A$ not $V_{\mathrm{f}} \mathrm{A}$.

5. Page 60, 15 th line up. Substitute A for $A$.

6. Page 67, equation 3.87. Substitute $K_{1}$ and $K_{2}$ for $k_{1}$ and $k_{2}$.

7. Page 85 , equation 4.55. For (3) read (4), and for (4) read (3).

8. Page 126, equation 5.57. In the last term of the equation substitute $\sigma_{\mathrm{A} 2 \mathrm{Q}} Q$ for $\sigma_{\mathrm{AQ}}^{2} Q$.

9. Page 166, equation 7.35. R.H.S. should read $\phi\left[1-\exp \left(-\varepsilon_{\lambda}^{\prime} c d\right)\right]$.

10. Page 166,6 th line up. Substitute $(O D \leqslant 0.05)$ for $(A \leqslant 0.05)$.

11. Page 170, equation 7.46. Extreme R.H.S. should read $k_{2} B$.

12. Page 174 , line between equations 7.59 and 7.60. For $\left(\bar{y}=v^{-1}\right)$ substitute $\left(\bar{y}=\bar{v}^{-1}\right)$.

13. Page 179, first part of equation 7.74. R.H.S. should read $\Sigma v^{3} / \Sigma v^{4}$.

14. Page 190, line following equation 8.36. Delete 'a'.

15. Page 199, line following equation 8.65. Substitute $\bar{v}$ for $v$.

16. Page 202, equation 9.3. For $\frac{\mathrm{d} v_{0}}{\mathrm{~d} A}$ substitute $\frac{\mathrm{d}^{2} v_{0}}{\mathrm{~d} A^{2}}$.

17. Page 219, equations 9.71 and 9.72. For $v$ substitute $\bar{v}$.

18. Page 229, line below equation 9.116. For $v_{\mathrm{T}}$ substitute $v_{\tau}$.

19. Page 233, last line. Insert ' $T$ ' between 'two' and 'protomers'. 


\section{Contents}

Preface $x i$

1 Enzymes as Biological Catalysts 1

1.1 The enzymes 1

1.1.1 Structure of enzymes 1

1.1.2 Flexibility of enzymes 3

1.1.3 Classification of enzymes 3

1.2 The substrates 4

1.2.1 Cofactors 4

1.3 The enzyme-substrate complex 6

1.3.1 The existence of the enzyme-substrate complex 6

$\begin{array}{lll}\text { 1.3.2 The active site } & 7\end{array}$

1.3.3 The function of the enzyme-substrate complex 8

1.3.4 The origin of the rate enhancement 11

1.4 Enzyme-modifier complexes 15

$\begin{array}{lll}1.4 .1 & \text { Inhibitors } & 15\end{array}$

$\begin{array}{ll}1.4 .2 \text { Activators } & 18\end{array}$

1.4.3 Allosteric effectors 18

$\begin{array}{ll}1.5 & \text { Mechanisms of enzyme catalysis } \\ 1 & 19\end{array}$

1.5.1 Representation of enzyme mechanisms 19

1.5.2 Classification of enzyme mechanisms 20

1.5.3 Examples of enzyme mechanisms 23

2 Introduction to the Kinetic Study of Enzymes 29

$2.1 \quad$ Nature of kinetic studies $\quad 29$

2.2 The kinetics of uncatalysed reactions 29

2.3 The origins of enzyme kinetics 30

2.4 The rate equation for a single substrate reaction 31

2.5 An examination of the assumptions employed in enzyme kinetics 33

2.5.1 The assumed mechanism 33

2.5.2 The steady state of equilibrium assumptions 34

2.5.3 The initial rate assumption 37

2.5.4 The assumption that the substrate concentration is much larger than the enzyme concentration 38

2.5.5 The implicit assumption that $K_{\mathrm{A}}$ and $V_{\mathrm{f}}$ can be accurately determined 
3.1 The single-substrate, single-product enzyme-catalysed reaction

3.1.1 Derivation of the Michaelis-Menten equation 43

3.1.2 Significance of the kinetic constants 44

3.1.3 The form of the Michaelis-Menten equation 45

3.1.4 The assay of enzyme concentration 45

3.2 Estimation of kinetic constants 46

3.2.1 The Lineweaver-Burk plot 47

3.2.2 The Hanes plot 48

3.2.3 The Eadie plot 48

3.2.4 The Eisenthal-Cornish-Bowden plot 49

3.2.5 Which is the best plot? $\quad 50$

3.3 The inhibition of enzyme activity 50

3.3.1 Competitive inhibition $(\alpha=\infty) \quad 51$

3.3.2 Partially competitive inhibition $(\infty>\alpha>1$ and $\beta=1)$

3.3.3 Non-competitive inhibition $(\alpha=1, \beta=0)$

3.3.4 Partially non-competitive inhibition $(\alpha=1,0<\beta<1)$

3.3.5 Mixed inhibition $(\infty>\alpha>0$, where $\alpha \neq 1$ and $\beta=0)$

3.3.6 Uncompetitive inhibition $\quad 55$

3.3.7 Substrate inhibition $\quad 57$

3.3.8 Product inhibition $\quad 57$

3.3.9 Irreversible inhibition $\quad 58$

3.3.10 Dixon plots $\quad 58$

3.4 Activation and two-substrate reactions $\quad 59$

3.4.1 The enzyme binds A and B in complex 60

3.4.2 The enzyme binds A and B in order 60

3.4.3 The enzyme binds A and B randomly 60

3.4.4 Aspects of substrate equilibria $\quad 62$

3.5 The effect of $\mathrm{pH} \quad 66$

3.5.1 The effect of $\mathrm{pH}$ on $V_{\mathrm{f}} \quad 67$

3.5.2 The effect of $\mathrm{pH}$ on the Michaelis constant 69

3.6 The effect of temperature $\quad 70$

3.6.1 The effect of temperature on $V_{\mathrm{f}} \quad 70$

3.6.2 The effect of temperature on $K_{\mathrm{A}} \quad 71$

3.7 The integrated Michaelis-Menten equation 71

3.7.1 The irreversible Michaelis-Menten reaction 72

$\begin{array}{ll}\text { 3.7.2 Later developments } & 72\end{array}$

4 Briggs-Haldane Kinetics, Derivation of Rate Equations 74

4.1 The King and Altman (1956) method 74

4.1.1 Application of determinants; Cramer's Rule 75

4.1.2 Determination of concentration of enzyme-intermediate 77

$\begin{array}{ll}\text { 4.1.3 Derivation of rate equations } & 79\end{array}$

4.2 Rate equations for enzyme mechanisms with three enzyme-intermediates 
4.3 Rate equations for enzyme mechanisms with four enzyme-intermediates

$\begin{array}{ll}\text { 4.3.1 Ordered } \mathrm{Bi} \mathrm{Bi} & 83\end{array}$

$\begin{array}{ll}\text { 4.3.2 Iso Theorell-Chance } & 84\end{array}$

$\begin{array}{lll}\text { 4.3.3 Ping Pong Bi Bi } & 84\end{array}$

4.3.4 Random Bi Uni $\quad 85$

4.4 Structural rules for graphical derivation of rate equations 86

4.5 The routine derivation of the enzyme distribution function for unbranched mechanisms

4.6 Determination of the distribution function for branched mechanisms by reducing the apparent number of enzymeintermediates

4.7 Determination of the enzyme distribution function for branched mechanisms using a slide rule

4.8 Determination of the rate equation using computers

4.9 Equilibrium and partial equilibrium mechanisms

4.9.1 Chemical equilibrium 98

4:9.2 Michaelis-Menten equilibrium 99

4.10 The effect of dead-end inhibition on the rate equations for unbranched mechanisms

\section{Briggs-Haldane Kinetics: Properties of Mechanisms with} Unbranched Catalytic Cycles

5.1 The experimental investigation of the rate equation 106

5.1.1 Determination of enzyme distribution function

5.2 Mechanistic interpretation of enzyme distribution function

5.2.1 Operational significance of the cyclic set nomenclature for unbranched mechanisms

5.2.2 Mechanistic origins of initial velocity and product inhibition patterns

5.2.3 The basis of complementarity in unbranched mechanisms

5.2.4 Determination of mechanism by factorisation of $\sigma$ constants

$\begin{array}{ll}\text { 5.2.5 Multiple binding by reactants } & 126\end{array}$

5.2.6 Dead-end inhibition

5.2.7 The range of unbranched mechanisms with distinct initial velocity and product inhibition patterns

5.3 Determination of enzyme mechanism: quantitative aspects

5.3.1 The Michaelean system of kinetic constants

5.3.2 Quantitative features of initial velocity data

5.3.3 Estimation of kinetic constants - a variant of the Dalziel procedure

5.3.4 The calculation of rate constants from kinetic constants

5.3.5 The detection of isomerisation reactions 
6 Briggs-Haldane Kinetics: Properties of Mechanisms with more than one Catalytic Cycle

6.1 The analysis of branched mechanisms by the Wong and Hanes (1962) procedure

6.1.1 The branched mechanisms of Wong and Hanes

6.1.2 The concept of degree: initial velocity studies as a function of substrate concentrations

6.1.3 The concept of degree: saturation velocity studies as a function of the second substrate concentration

6.1.4 The concept of degree: saturation studies as a function of a product concentration

6.1.5 The concept of degree: alternative cycles linked through single enzyme-intermediates

6.1.6 Limitations of the concept of degree

6.2 The investigation of unbranched mechanisms by the simultaneous catalysis of two reactions with alternative substrates

7 Measurements of the Initial Rates of Enzyme-Catalysed Reactions and Estimation of the Parameters of Reaction

7.1 The design of an enzyme kinetic experiment 156

7.1.1 Determination of preliminary constants 156

$\begin{array}{ll}\text { 7.1.2 The choice of reactant concentrations } & 160\end{array}$

7.1.3 The method of determining the initial rate 162

7.1.4 The attainment of equilibrium 163

7.1.5 The concentration of enzyme required to give a convenient initial rate 164

7.2 Methods of determining the initial rate 165

7.2.1 Spectrophotometric methods 165

7.2.2 Fluorimetric methods 166

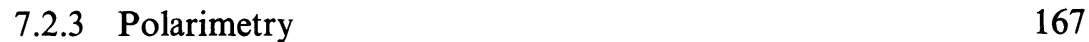

$\begin{array}{ll}\text { 7.2.4 Methods employing electrodes } & 167\end{array}$

$\begin{array}{ll}\text { 7.2.5 Chromatographic methods } & 167\end{array}$

7.2.6 Estimation of the initial rate 168

$\begin{array}{lll}7.3 & \text { Coupled enzyme assays } & 169\end{array}$

$\begin{array}{ll}\text { 7.3.1 Coupled assay with one auxiliary enzyme } & 170\end{array}$

7.3.2 Coupled assays with more than one auxiliary enzyme 171

$\begin{array}{lll}7.4 & \text { Error in enzyme kinetic data } & 172\end{array}$

7.4.1 The weighting of enzyme kinetic data 173

7.5 The graphical analysis of kinetic data 175

7.5.1 Comparison of methods of graphical analyses 176

7.5.2 Distribution of datum points 177

$\begin{array}{lll}\text { 7.6 The statistical analysis of kinetic data } & 178\end{array}$

7.6.1 The least squares method 178

$\begin{array}{ll}\text { 7.6.2 The direct linear plot } & 179\end{array}$ 
8 Isotope Transfer at Equilibrium 182

8.1 The experimental method 182

$\begin{array}{ll}\text { 8.2 The interpretation of results } & 183\end{array}$

8.3 Derivation of rate equations for isotope transfer at equilibrium

8.3.1 Cleland's method (1970) 186

$\begin{array}{ll}\text { 8.3.2 The one-system method } & 187\end{array}$

$\begin{array}{lll}\text { 8.3.3 The two-system method } & 188\end{array}$

$\begin{array}{ll}\text { 8.3.4 The equilibrium assumption } & 190\end{array}$

8.4 Rate equations for isotope exchange at equilibrium in

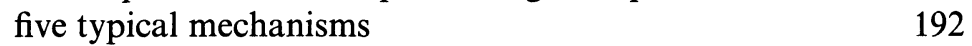

8.4.1 The Ordered Bi Bi reaction 192

8.4.2 The Iso-Theorell-Chance reaction 195

8.4.3 The Ping Pong Bi Bi reaction 197

8.4.4 The Random Order Bi Bi reaction 198

8.4.5 The relative magnitudes of the rates of transfer and 199

8.4.6 The equilibrium random order $\mathrm{Bi} \mathrm{Bi}$ reaction 200

$\begin{array}{ll}\text { 8.4.7 Dead-end inhibition } & 201\end{array}$

$\begin{array}{lll}\text { 8.4.8 Summary } & 201\end{array}$

9 The Kinetics of Allosteric Enzymes 202

9.1 The properties of higher degree rate equations 202

$\begin{array}{ll}\text { 9.1.1 Hill plots } & 204\end{array}$

$\begin{array}{lll}9.2 & \text { Regulatory enzymes } & 208\end{array}$

9.3 The physical basis of higher degree rate equations 211

$\begin{array}{lll}9.4 & \text { Independent binding } & 213\end{array}$

9.4.1 The binding of one ligand by a protomer with identical

9.4.2 The binding of several ligands by a protomer with identical and independent binding sites 216

9.4.3 The binding of ligands by a protomer with different but independent binding sites

9.4.4 Summary of independent binding - extension to oligomers 222

$\begin{array}{lll}9.5 & \text { Co-operative binding } & 222\end{array}$

9.5.1 The Adair hypothesis 222

9.5.2 A general, two-state conformational model for co$\begin{array}{ll}\text { operative binding } & 225\end{array}$

9.5.3 The sequential models of Koshland, Némethy and Filmer 233

9.5.4 The concerted model of Monod, Wyman and Changeux 236

9.6 The binding of ligands by a polymerising protein system 240

9.7 Summary 243

Appendix: Design of a Slide Rule for Deriving the Rate Equations of Enzyme-catalysed Reactions with Unbranched 245 Mechanisms 


\section{Preface}

'Kinetics deals with the rate of chemical reaction, with all the factors which influence the rate of reaction and with the explanation of the rate in terms of the reaction mechanism' (Frost and Pearson). This succinct definition of chemical kinetics applies with equal validity to the study of the initial rates of enzyme-catalysed reactions, the subject matter of this book. It indicates the two main reasons why biochemists are interested in enzyme kinetics. First, kinetics provides the most definitive method of determining the mechanism of reaction, in the sense that any conclusion about the mechanism (which can, of course, depend on non-kinetic experiments) must be consistent with the kinetic behaviour of the enzyme; and second, the mechanism, and its associated constants, allows the biochemist to discuss the behaviour of the enzyme in a cellular environment more meaningfully and, at a more practical level, to devise efficient assays either for, or incorporating, the enzyme.

This book is almost exclusively concerned with the first function of enzyme kinetics, namely to define the mechanism of reaction. It will be noted that the method adopted is essentially negative; proposed mechanisms of reaction can be excluded as inconsistent with the kinetic data, but consistent mechanisms cannot be distinguished. The role of the enzyme kineticist is, therefore, to postulate mechanisms and by analysis predict their behaviour. The licence that is thereby provided to the rational imagination is at once the chief attraction and the principal danger of the method, for science in general, and biochemistry in particular, is seldom rational except by hindsight and the kineticist must be continually reminded of the complexity and unpredictability of enzyme behaviour. The attraction remains the satisfaction of creating working models and, under its compulsion, there has grown up a large body of enzyme kinetic theory. Formidable kinetic problems lie ahead in biochemistry not only to do with individual enzymes but also with the co-operative activity of groups of enzymes. Enzyme kinetic theory is therefore an essential part of the undergraduate curriculum, both explaining what is known and providing the necessary springboard for further investigation of enzyme activity.

It is true that undergraduates commonly regard enzyme kinetics as a difficult subject, largely because of its mathematical content. But it is my conviction, gained from teaching the subject for 10 years, that the mathematical description of kinetic ideas cannot be circumvented without delaying or even preventing the understanding of those ideas. And further, that most undergraduates come to realise, with a little practice, that the application of mathematics in steady-state enzyme kinetics is not intrinsically difficult but merely time-consuming. Indeed, at least $90 \%$ of the equations in this book can be derived with only the most elementary grounding in algebraic manipulation. Whether this simplicity will be retained as the student moves forward is an open question, but one, fortunately, that is not relevant here. 
For the present, I have given expression to my conviction by developing kinetic ideas mathematically; I have, however, presented the argument as fully as possible so that the reader can pass from one stage to the next with the minimum of difficulty. In this connection, I should like to advise the reader to equip himself with pencil and paper; the actual experience of manipulating equations is invaluable, more, it is inescapable if real progress is to be made.

Another point arises here concerning the presentation. I have not attempted to give an exhaustive account of enzyme kinetics (indeed, a treatment of pre-steady zstate kinetics is one notable omission); what I have attempted to do is to identify some of the basic ideas of steady-state kinetics and to explain them in a way that will enable the reader to apply the general principle involved to a particular problem that engages his attention. For this reason, the book should also be helpful to graduate students about to embark on kinetic studies themselves.

The layout of the book can be seen in the chapter headings and requires little comment. Chapter 1 is an introduction to the physical basis of enzyme action and provides examples of enzyme mechanisms that are discussed in detail elsewhere in the book. Chapters 2-8 deal with the kinetic behaviour of simple enzymes; in particular, Chapter 7 may be noted for its emphasis on practical considerations and Chapter 8 for its treatment of isotope transfer between the reactants and products of the enzyme-catalysed reaction. The last chapter describes the kinetic behaviour of oligomeric enzymes, a group of enzymes that are especially important for the regulation of metabolic activity.

Finally, thanks are due to Professor Walter Bartley and to my colleagues, notably Elizabeth Heyde, William Ferdinand, Neil Macfarlane and Julian Kinderlerer, for their help in discussing the problems of enzyme kinetics. I also remember many other friends and colleagues who have helped me, often very substantially; this book could not have been written without them. 